\title{
Primary Ovarian Pregnancy after Interval Tubal Ligation- An Unexpected Conception at an Unusual Location
}

\author{
Madhavi Omdeorao Musale, Prashanth Rudrappa, Udaya Kumar M and Ramaswamy Anikode Subramanian* \\ Department of Pathology PES Institute of Medical Sciences and Research, Kuppam. Andhra Pradesh, INDIA
}

\section{ABSTRACT}

Ovarian pregnancy constitutes $<3 \%$ of all ectopic pregnancies with an incidence ranging from 1:6,000 to 1:40,000 pregnancies. Ovarian ectopic pregnancy is a very rare occurrence after the interval tubal ligation.

A 25 year old multi gravida, post tubectomised woman presented with acute lower abdominal pain with history of interval sterilisation after 4 months of last child birth. Ultrasonography detected a seven weeks gestation with cardiac activity in right adnexa with no evidence of intrauterine pregnancy. Right salpingo-oopherectomy with removal of an unruptured pregnancy from the right ovary was done by exploratory laparotomy. On histopathological examination, chorionic villi within the ovarian stroma was seen.

Though ovarian pregnancy is rare, one has to consider the possibility of an ectopic gestation even in post interval tubal-ligation cases. This would help in early diagnosis and reduce the rate of maternal morbidity and mortality.

\section{Keywords: Ovarian pregnancy, tubal ligation.}

\section{Introduction}

Ectopic pregnancy is an important health problem and accounts for $10 \%$ of all maternal mortality. [1] Ovarian pregnancy is a rare event occurring in $1-3 \%$ of all ectopic pregnancies. [2] Definite diagnosis of primary ovarian ectopic pregnancy is critical and also very difficult due to non specific clinical presentations. To resolve this problem, the von Spiegelberg criteria is used. According to this criteria, diagnosis of primary ovarian ectopic pregnancy should be considered when all the following are present:

1. The ectopic ovarian pregnancy is attached to uterus by ovarian ligament.

2. The gestational sac is seen within ovary grossly.

3. The tube on involved side is intact.

4. On histology gestational sac is seen within the ovarian tissue. [3]

\section{Case report}

We hereby report a case of 25 year old multigravida woman who presented with ovarian ectopic pregnancy after interval tubal ligation. Patient had presented with acute lower abdominal pain with history of seven months of amenorrhea. She had previous two full term caesarean deliveries, followed by interval tubectomy after 4 months of last child birth.

Ultrasonography detected a seven weeks gestation with cardiac activity in right adnexa. There was no evidence of intrauterine pregnancy. Right salpingo-oopherectomy was done by exploratory laparotomy. Intraoperatively uterus and both tubes were found normal with enlarged right sided ovary.

The specimen of the right ovary measuring $5 \times 3.5 \times 2 \mathrm{~cm}$ with part of fallopian tube measuring $4 \mathrm{~cm}$ in length was received in the department of pathology.

The surface of ovary was congested and cystic. The cutsection of the ovary showed embryo, multiple small cystic spaces and hemorrhagic areas.

Histopathological sections from the ovary showed multiple chorionic villi lined by trophoblastic tissue within ovarian stroma. The sections from the fallopian tube showed no evidence of gestational tissue. This finding fulfilled the Spiegelberg's criteria. Hence a diagnosis of right-sided ovarian pregnancy was made.

\section{Discussion}

Primary ovarian pregnancy is one of the rarest types of extra-uterine pregnancy. Ectopic pregnancy is the most important cause of maternal death in the first trimester accounting for approximately $10 \%$ of deaths related to pregnancy [4]. The exact incidence of ovarian pregnancy is unknown because in large number of asymptomatic patients the conceptus dies and involutes spontaneously [1]. Younger age, endometriosis, pelvic inflammatory disease (PID), intra-uterine devices, use of ovulatory medications and assisted reproductive techniques are a few risk factors that have contributed to the increased incidence of ovarian 


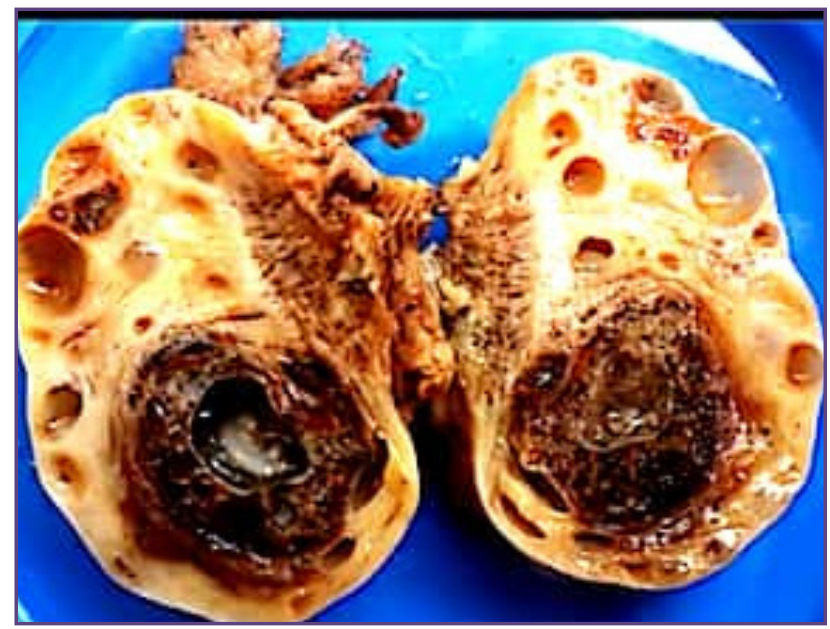

Fig.1: Gross: Embryo within excised right ovary.

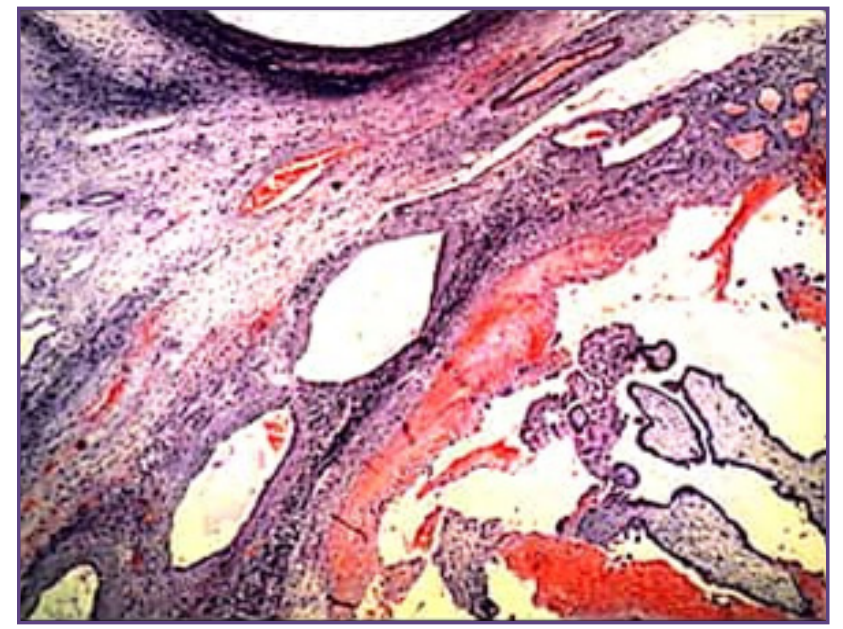

Fig. 2: Chorionic villi within ovarian stroma (H\&E stain, 100X).

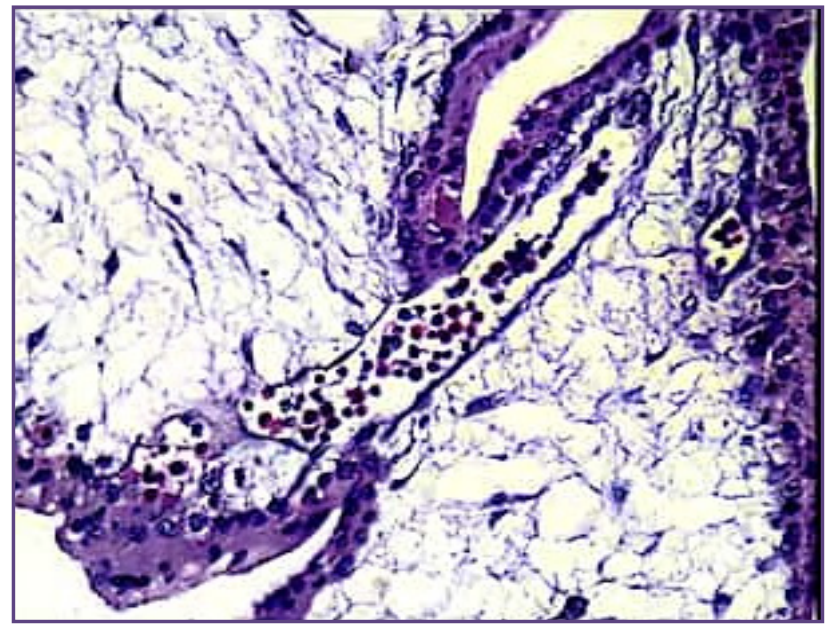

Fig. 3: Edematous stroma of villi and nucleated embryonic RBCs within blood vessel (H\&E stain, 400X).

pregnancy [5].PID can result in a reduction of tubal motility and/or a thickening of the ovarian albuginea secondary to the inflammatory response, which can cause a reduction of follicular dehiscence and a subsequent increased risk of intra-follicular pregnancy [6].Ectopic gestation after tubal ligation is due to recanalization or formation of tuboperitoneal fistula, in which spermatozoa may pass through, but the fertilised ovum fails to go through, so implantation occurs in distal tubal segment [7][8]. In the present case, the patient did not have any of the risk factors described above. Wittich et al had reported a case of ovarian ectopic pregnancy following postpartum tubal sterilisation which can occur due to oedematous, congested and friable tubes resulting in incomplete occlusion of tubal lumen [9]. In our case ovarian pregnancy following interval tubal ligation is a rare occurrence and only few cases have been reported till date.

\section{Conclusion}

This is an example of a rare diagnostic entity at an uncommon site with unusual presentation. Though ovarian pregnancy is rare, one has to consider the possibility of an ectopic gestation even in post interval tubal-ligation cases. This would help in early diagnosis and reduce the rate of maternal morbidity and mortality.

\section{Acknowledgement}

We sincerely thank the department of Obstetrics and Gynaecology, for the kind co-operation extended to us for the work up of the case.

\section{References}

1. Das S, Kalyani R, Lakshmi V, Kumar MH. Ovarian pregnancy. Indian journal of Pathology and Microbiology. 2008.1;51(1):37. 
2. Roy J, Sinha B. A Ovarian pregnancy: Two case reports. AMJ. 2013:406-410.

3. Spiegelberg O. Zuv casuistic der ovarial Schwangerschft. Arch Gynackol 1978;13:73-6.

4. Singh S, Alwani M, Malukani K. Ruptured Primary Ovarian Ectopic Pregnancy: A rare case report. Int J Reprod Contracept Obstet Gynecol 2015;4:480-2.

5. Fox H. Ectopic pregnancy. In: Fox H, Wells M editors. Haines and Taylor obstetrical and gynecological pathology. Vol 2. 4th ed. New York: Churchill Livingstone; 1995. p. 1128-9.
6. Roy J, Babu AS. Ovarian pregnancy: Two case reports. The Australasian medical journal. 2013;6(8):406.

7. Stock RJ, Nelson KJ. Ectopic pregnancy subsequent to sterilization:histologic evaluation and clinical implications. Fertil Steril 1984;42:211-5.

8. Davis MR. Reccurent ectopic pregnancy after tubal sterilization. Obstet Gynecol 1986 Sep;68(3suppl):44S-45S.

9. Wittich AC. Primary ovarian preg after postpartum bilateral tubal ligation: a case report. J Family Reprod Med 2004 Sep, 49(9):759-761.

*Corresponding author:

Dr. A S Ramaswamy, 52, Vignesh Nilayam, 11th Cross, T C Palya, K R Puram, Bangalore 560036 INDIA

Phone: +919493726494

Email: dr_Asr@Rediffmail.Com

Financial or other Competing Interests: None. 